\title{
Identification of crucial genes and pathways associated with colorectal cancer by bioinformatics analysis
}

\author{
XIAOQUN LIU $^{1 *}$, XIANGDONG LIU $^{2 *}$, TIANKUI QIAO ${ }^{1}$ and WEI CHEN ${ }^{1}$ \\ ${ }^{1}$ Center for Tumor Diagnosis and Therapy; ${ }^{2}$ Department of Ophthalmology, \\ Jinshan Hospital, Fudan University, Shanghai 201508, P.R. China
}

Received May 10, 2019; Accepted October 22, 2019

DOI: $10.3892 / \mathrm{ol} .2020 .11278$

\begin{abstract}
Colorectal cancer (CRC) is a prevalent malignant tumour type arising from the colon and rectum. The present study aimed to explore the molecular mechanisms of the development and progression of CRC. Initially, differentially expressed genes (DEGs) between CRC tissues and corresponding non-cancerous tissues were obtained by analysing the GSE15781 microarray dataset. The Database for Annotation, Visualization and Integrated Discovery was then utilized for functional and pathway enrichment analysis of the DEGs. Subsequently, a protein-protein interaction (PPI) network was created using the Search Tool for the Retrieval of Interacting Genes and Proteins database and visualized by Cytoscape software. Furthermore, CytoNCA, a Cytoscape plugin, was used for centrality analysis of the PPI network to identify crucial genes. Finally, UALCAN was employed to validate the expression of the crucial genes and to estimate their effect on the survival of patients with colon cancer by Kaplan-Meier curves and log-rank tests. A total of 1,085 DEGs, including 496 upregulated and 589 downregulated genes, were screened out. The DEGs identified were enriched in various pathways, including 'metabolic pathway', 'cell cycle', 'DNA replication', 'nitrogen metabolism', 'p53 signalling' and 'fatty acid degradation'. PPI network analysis suggested that interleukin-6, MYC, NOTCH1, inhibin subunit $\beta$ A (INHBA), CDK1, cyclin (CCN)B1 and CCNA2 were crucial genes, and their expression levels were markedly upregulated. Survival analysis suggested that upregulated INHBA significantly decreased the survival probability of patients with CRC. Conversely, upregulation of CCNB1 and CCNA2 expression levels were associated with increased survival probabalities. The identified DEGs,
\end{abstract}

Correspondence to: Dr Tiankui Qiao, Center for Tumor Diagnosis and Therapy, Jinshan Hospital, Fudan University, 1508 Longhang Road, Jinshan, Shanghai 201508, P.R. China

E-mail: 18019206896@163.com

*Contributed equally

Key words: differentially expressed genes, colorectal cancer, bioinformatics analysis particularly the crucial genes, may enhance the current understanding of the genesis and progression of CRC, and certain genes, including INHBA, CCNB1 and CCNA2, may be candidate diagnostic and prognostic markers, as well as targets for the treatment of CRC.

\section{Introduction}

Colorectal cancer (CRC) is a serious threat to human health; it is one of the most common malignancies and the fourth leading cause of cancer-associated mortality worldwide (1). According to the GLOBOCAN 2018 database, 1.8 million newly-diagnosed cases of CRC and 861,600 cases of CRC-associated mortality were recorded worldwide, in the same year (2). Despite advances in the available therapeutic strategies, the clinical outcomes for CRC remain far from satisfactory due to cancer recurrence, metastasis, and resistance to radio- and chemotherapy $(3,4)$. Therefore, further investigations into the precise molecular mechanisms that account for colorectal carcinogenesis and CRC progression are of great significance and are urgently required; this may provide vital clues for the identification of novel diagnostic and therapeutic targets and monitoring disease progression.

$\mathrm{CRC}$ is considered to be a highly heterogeneous disease mainly caused by interactions between genetic alterations and environmental factors (5). Several genes and cellular signalling pathways, such as receptor for activated C kinase 1 (RACK1) and long non-coding RNA breast cancer anti-estrogen resistance 4 (lncRNA BCAR4), have been reported to serve important roles in the occurrence and development of CRC (6-8). For instance, the expression of RACK 1 has been reported to be significantly upregulated in CRC tissues compared with in adjacent normal tissues (6). In vitro, overexpression of RACK1 markedly promotes cellular proliferation, migration and invasion (7). In addition, it has been reported that IncRNA BCAR4 is closely associated with $\mathrm{CRC}$ initiation and dissemination through targeting microRNA (miR)-665/STAT3 signalling (8). Despite these thorough and detailed studies (6-8) to identify novel targets for CRC management, to the best of our knowledge, a comprehensive presentation of the crucial key genes and signalling pathways implicated in CRC is lacking.

Gene microarray profile analysis, a high-throughput method for detection of mRNA expression in tissues, has increasingly become a promising tool in medical oncology. 
By analysing differential gene expression between tumour tissues and normal control tissues, an improved understanding of the molecular pathogenesis of various cancer types may be attained, facilitating the identification of potential target genes and signalling pathways for precision therapy (9). In previous decades, numerous studies on gene expression profiles in cancer have used microarray technology $(10,11)$, but only one study has focused on CRC (12). In addition, comparative analysis of differentially expressed genes (DEGs) remains relatively limited (13). Furthermore, reliable biomarker profiles for discriminating CRC from normal tissues require further identification. In addition, the interactions among the DEGs identified, particularly the interaction networks and important signalling pathways, should be elucidated.

In the present study, data were extracted from the Gene Expression Omnibus (GEO) database. Next, the DEGs between CRC tissues and the corresponding non-cancerous tissues were screened. The possible functions of, and potential pathways enriched by, the DEGs were then predicted by enrichment analysis. Furthermore, protein-protein interaction (PPI) networks were generated, and centrality analysis was performed to identify the crucial genes that were potentially involved in the development of CRC. In addition, the expression levels of the crucial genes and their effect on the survival of patients with CRC were further evaluated using UALCAN. Based on integrated bioinformatics analysis of gene expression, the present study aimed to further elucidate the molecular pathogenesis of CRC and identify reliable diagnostic and prognostic biomarkers as well as therapeutic targets.

\section{Materials and methods}

Microarray data. The raw microarray data of the GSE15781 dataset, contributed by Snipstad et al (14), were downloaded from the GEO database (http://www.ncbi.nlm.nih.gov/geo/), a public functional genomics data repository containing arrayand sequence-based data. The dataset included 10 locally advanced CRC tissues and the corresponding non-cancerous tissues. The data were pre-processed with Agilent GeneSpringGX software (version 11.5; Agilent Technologies, Inc.) using the Robust Multichip Averaging algorithm (15). The probe set IDs were converted into the corresponding gene symbols according to the annotation information derived from the GPL2986 platform (ABI Human Genome Survey Microarray version 2.0) in the GEO database. In the event of various probe sets corresponding to the same gene, the mean expression values of those probe sets were obtained.

Identification of DEGs. Agilent GeneSpringGX software was further utilized to screen DEGs. Significance analysis of the expression of genes between each pair of cancerous and normal tissues was jointly implemented by a paired t-test and fold change (FC) calculation. The Benjamini and Hochberg method (16) was then used to calculate the adjusted P-value. A $\left|\log _{2}(\mathrm{FC})\right|$ value of $\geq 1$ and an adjusted $\mathrm{P}$-value of $<0.05$ were considered to be the cut-off criteria for the identification of DEGs. In addition, to categorize the data into two groups of different expression patterns, hierarchical clustering analysis was applied in R language (version 3.5.3; https://www.r-project.org/) using the gplots package (version 3.0.1; https://cran.r-project.org/web/packages/gplots/).

Gene Ontology (GO) and pathway enrichment analysis of DEGs. The Database for Annotation, Visualization and Integrated Discovery (DAVID; version 6.8; https://david. ncifcrf.gov) is an essential online tool used for systematically automating the processes of biological term classification and enrichment analysis of gene clusters (17). In the present study, to categorize the DEGs and the enriched pathways, the DAVID database was employed to identify and visualize the GO (www.geneontology.org) terms and Kyoto Encyclopedia of Genes and Genomes (KEGG; www.genome.jp) pathways enriched by the DEGs. Enrichment by $\geq 10$ genes and $\mathrm{P}<0.05$ were set as the cut-off criteria for significant enrichment. The $\mathrm{P}$-value for each enriched pathway was calculated via $-\log _{10}$ transformation. $\mathrm{P}<0.05\left[-\log _{10}(\mathrm{P}\right.$-value $\left.)>1.30\right]$ was considered to indicate statistical significance.

Analysis of the PPI network of the DEGs. The Search Tool for the Retrieval of Interacting Genes and proteins (STRING) database (version 10.0; string-db.org) encompasses $>9,000,000$ proteins and integrates $>932,000,000$ known and predicted interactions between proteins from a large number of organisms, including Homo sapiens (18). In the present study, the STRING database was used to construct the predicted PPI network of DEGs with a minimum required interaction score of 0.4. The PPI network was then visualized using Cytoscape software (version 3.6.0) (19). Subsequently, CytoNCA (version 2.1.6), a Cytoscape plugin for centrality analysis of protein interaction networks, was utilized to identify crucial nodes (genes) in the network (20). In the present study, the crucial genes were screened based on four different centrality measures: i) Eigenvector centrality; ii) degree centrality; iii) betweenness centrality; and iv) closeness centrality. According to the centrality values of the genes in the PPI network, the top 3 ranked genes were identified as the crucial genes.

Association between the expression levels of the crucial genes and the survival of patients with CRC. UALCAN is a user-friendly, interactive web resource allowing researchers to analyse cancer transcriptome data from The Cancer Genome Atlas (TCGA) database (https://portal.gdc.cancer.gov/) rapidly and easily (21). UALCAN is a vital portal for facilitating tumour gene expression and survival analyses using TCGA level 3 RNA-sequencing and clinical data from 31 types of cancer (22). UALCAN is designed to provide: i) Easy access to cancer transcriptome data available to the public; ii) high-quality graphs depicting gene expression and patient survival information based on gene expression; and iii) additional information regarding the selected genes/targets using links to databases, including GeneCards (https://www.genecards.org/), PubMed (https://www.ncbi.nlm.nih.gov/pubmed), TargetScan (https://www.targetscan.org/) and DrugBank (https://www. drugbank.ca/). In the present study, UALCAN was utilized to further validate and estimate the effect of the expression levels of the crucial genes on the survival of patients with colon cancer by drawing Kaplan-Meier curves and performing log-rank tests. Furthermore, the protein expression of the crucial genes was also determined by immunohistochemical 

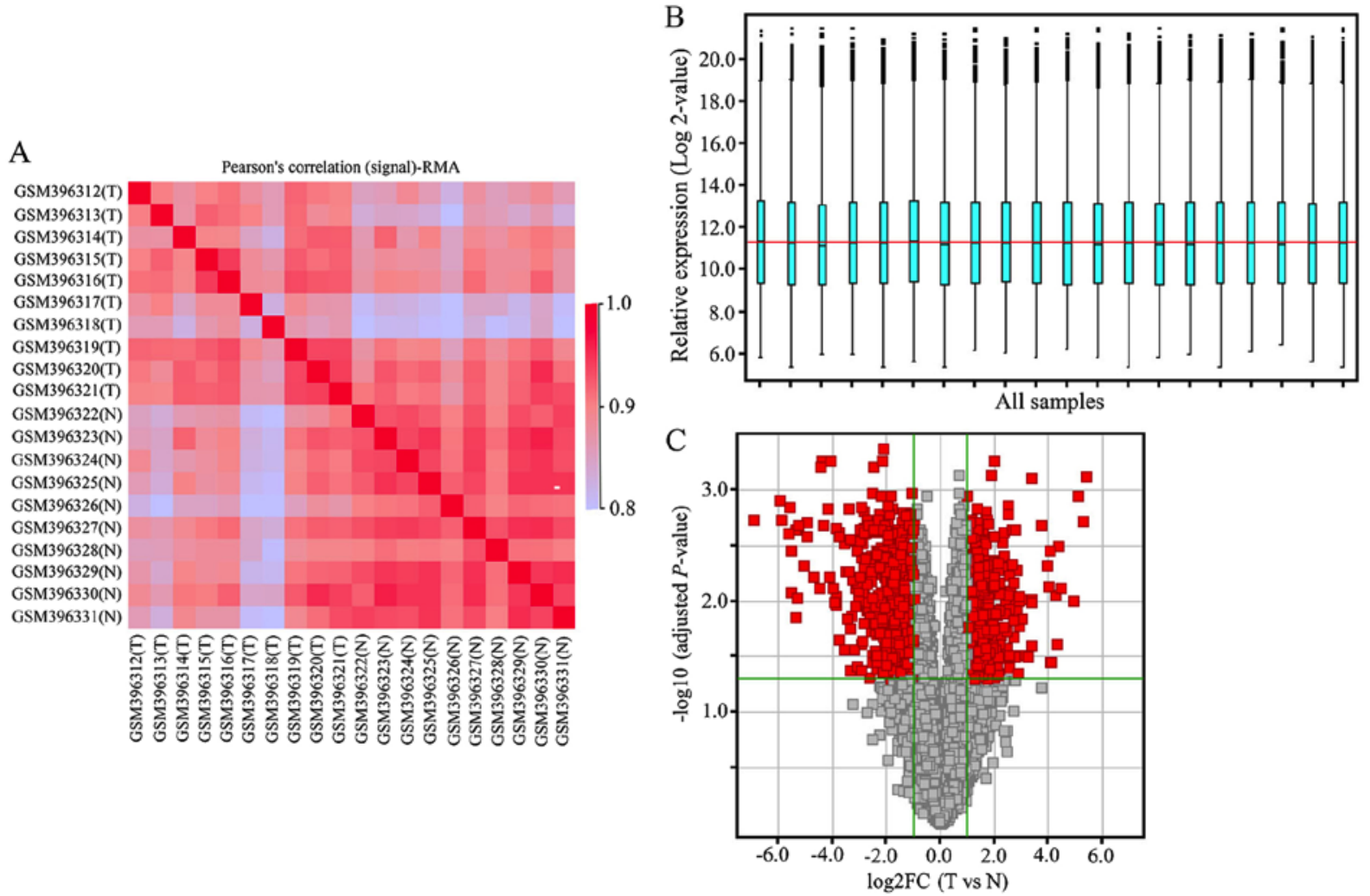

Figure 1. Identification of DEGs. (A) Pearson's correlation (signal) map. The correlation coefficient was close to 1.0, indicating high repeatability or similar distribution. The key on the right indicates the correlation coefficient. (B) Relative signal boxplot map. The red line is the baseline; a more similar distribution implies higher repeatability of the data. (C) Volcano plot comparing all DEGs. The red squares represent DEGs that were significant at llog 2 (FC) $\geq 1$. The red squares on the upper left and upper right represent down- and upregulated genes in colorectal cancer, respectively. T, tumour tissues; N, normal tissues; DEG, differentially expressed gene; FC, fold change; RMA, Robust Multichip Averaging.

staining analysis, which was obtained from the Human Protein Atlas (HPA) database (www.proteinatlas.org).

\section{Results}

Identification of DEGs. Initially, data from a total of 42 chips were acquired from GEO dataset GSE15781. Following quality control, 20 chips that included data from 10 CRC tissues and the corresponding matched normal tissues were selected. As shown in Fig. 1A and B, the Pearson's correlation (signal) map and relative signal boxplot map of the pre-treated data present the performance of normalisation. The data series from each chip were analysed separately and information on the expression levels of 16,227 genes was obtained using the GPL2986 platform. A total of 1,085 DEGs (tumour vs. normal tissues), including 496 upregulated and 589 downregulated genes, were selected based on the criteria of an adjusted P-value $<0.05$ and $\log _{2}$ (FC) $\mid \geq 1$ (Fig. 1C). As presented in Table I, the top 10 upregulated DEGs were matrix metalloproteinase (MMP)7, defensin $\alpha$ (DEFA)6, fatty acid binding protein 6 , keratin 23 , $\mathrm{C}-\mathrm{X}-\mathrm{C}$ motif chemokine ligand (CXCL) 8 , inhibin subunit $\beta$ A (INHBA), DEFA5, transcobalamin 1, regenerating family member $3 \alpha$ and MMP3, and the top 10 downregulated DEGs were aquaporin 8 , carbonic anhydrase 1 (CA1)2, insulin-like 5, guanylate cyclase activator $2 \mathrm{~A}$, caspase recruitment domain family member 14 , solute carrier family 26 member 3 , membrane spanning 4-domains A12, peptide YY, chloride channel accessory 4 and CA4.
Hierarchical clustering analysis of DEGs. Following the extraction of the expression values for the DEGs, hierarchical clustering analysis was performed. As presented in Fig. 2, the 20 specimens were divided into the CRC and normal groups. The heatmap indicated that, in comparison with normal tissues, CRC tissues exhibited more downregulated than upregulated genes. These results indicated that the DEGs exhibited distinct expression patterns in tumour and normal tissues.

GO term enrichment analysis of DEGs. To investigate the function of the DEGs, GO term enrichment analysis was performed using the online tool DAVID. The analysis indicated that the DEGs were significantly enriched in $93 \mathrm{GO}$ terms, including 54 terms in the category biological process (BP), 12 terms in the category molecular function (MF) and 27 terms in the category cellular component (CC) (Table SI). The top 5 BP terms were 'mitotic nuclear division', 'positive regulation of cell proliferation', 'one-carbon metabolic process', 'cell division' and 'immune response'. The top 5 MF terms were 'hormone activity', 'sodium channel regulator activity', 'NAD binding', 'protein homodimerization activity' and 'protein binding'. The top 5 CC terms were 'extracellular space', 'extracellular exosome', 'extracellular region', 'apical plasma membrane' and 'cytosol' (Table II). In particular, the upregulated DEGs were mainly enriched in the terms 'nucleoplasm' $\left(\mathrm{P}=6.32 \times 10^{-17}\right)$, 'mitotic nuclear division' $\left(\mathrm{P}=4.25 \times 10^{-14}\right)$ and 'cell division' $\left(\mathrm{P}=1.62 \times 10^{-12}\right)$ (Table SII). The downregulated DEGs were mainly enriched in the terms 'extracellular 
Table I. Top 10 upregulated and downregulated genes in colorectal cancer tumor vs. normal tissues.

\begin{tabular}{lcll}
\hline Gene symbol & $\begin{array}{c}\mathrm{Log}_{2} \\
\text { FC }\end{array}$ & $\begin{array}{c}\text { Adjusted } \\
\text { P-value }\end{array}$ & Expression \\
\hline MMP7 & 5.39 & $7.39 \times 10^{-4}$ & $\mathrm{Up}$ \\
DEFA6 & 5.33 & $1.90 \times 10^{-3}$ & $\mathrm{Up}$ \\
FABP6 & 5.25 & $1.10 \times 10^{-3}$ & $\mathrm{Up}$ \\
KRT23 & 5.24 & $9.90 \times 10^{-3}$ & $\mathrm{Up}$ \\
CXCL8 & 5.06 & $7.60 \times 10^{-3}$ & $\mathrm{Up}$ \\
INHBA & 5.05 & $3.20 \times 10^{-3}$ & $\mathrm{Up}$ \\
DEFA5 & 4.97 & $2.40 \times 10^{-2}$ & $\mathrm{Up}$ \\
TCN1 & 4.96 & $8.60 \times 10^{-3}$ & $\mathrm{Up}$ \\
REG3A & 4.92 & $3.50 \times 10^{-2}$ & $\mathrm{Up}$ \\
MMP3 & 4.04 & $3.50 \times 10^{-2}$ & $\mathrm{Up}$ \\
AQP8 & -6.90 & $1.80 \times 10^{-3}$ & Down \\
CA1 & -5.92 & $1.20 \times 10^{-3}$ & Down \\
INSL5 & -5.90 & $1.80 \times 10^{-3}$ & Down \\
GUCA2A & -5.61 & $2.40 \times 10^{-3}$ & Down \\
CARD14 & -5.59 & $1.40 \times 10^{-3}$ & Down \\
SLC26A3 & -5.54 & $3.50 \times 10^{-3}$ & Down \\
MS4A12 & -5.50 & $8.10 \times 10^{-3}$ & Down \\
PYY & -5.40 & $2.30 \times 10^{-3}$ & Down \\
CLCA4 & -5.39 & $1.40 \times 10^{-2}$ & Down \\
CA4 & -5.33 & $9.30 \times 10^{-3}$ & Down \\
\hline
\end{tabular}

FC, fold change; MMP7, matrix metalloproteinase-7; DEFA6, defensin alpha 6; FABP6, fatty acid binding protein 6; KRT23, keratin 23; CXCL8, C-X-C motif chemokine ligand 8; INHBA, inhibin $\beta A$; DEFA5, defensing alpha 5; TCN1, transcobalamin 1; REG3A, regenerating islet-derived 3 alpha; MMP3, matrix metalloproteinase-3; AQP8, aquaporin 8; CA1, carbonic anhydrase 1; INSL5, insulin-like 5; GUCA2A, guanylate cyclase activator 2a; CARD14, caspase recruitment domain family member 14; SLC26A3, solute carrier family 26 member 3; MS4A12, membrane-spanning 4-domains subfamily A member 12; PYY, peptide YY; CLCA4, chloride channel accessory 4; CA4, carbonic anhydrase 4.

exosome' $\left(\mathrm{P}=7.97 \times 10^{-20}\right)$, 'extracellular space' $\left(\mathrm{P}=2.62 \times 10^{-13}\right)$ and 'plasma membrane' $\left(\mathrm{P}=3.11 \times 10^{-9}\right)$ (Table SIII).

KEGG pathway enrichment analysis of DEGs. Furthermore, KEGG pathway analysis was performed to identify pathways in which the DEGs were involved. The results indicated that the DEGs were significantly enriched in 43 pathways, including 22 and 18 significantly enriched pathways for the upregulated and downregulated genes, respectively. The top 10 enriched pathways were the 'pentose and glucuronate interconversion', 'metabolic pathways', 'cell cycle', 'cytokine-cytokine receptor interaction', 'sulfur metabolism', 'DNA replication', 'nitrogen metabolism', 'p53 signalling pathway', 'fatty acid degradation' and 'pyrimidine metabolism' (Fig. 3). In particular, the most significant KEGG pathways enriched by the upregulated DEGs were 'cell cycle' $\left(\mathrm{P}=2.41 \times 10^{-8}\right)$, 'p53 signaling pathway' $\left(\mathrm{P}=2.71 \times 10^{-5}\right)$ and 'tumour necrosis factor signaling pathway' $\left(\mathrm{P}=3.46 \times 10^{-4}\right)$ (Table SIV). The most significant KEGG pathways enriched by the downregulated DEGs were 'retinol metabolism' $\left(\mathrm{P}=5.15 \times 10^{-4}\right)$, 'drug metabolism-cytochrome $\mathrm{P} 450$ ' $\left(\mathrm{P}=8.10 \times 10^{-4}\right)$ and 'metabolic pathways' $\left(\mathrm{P}=2.39 \times 10^{-3}\right)$ (Table SV).

Construction of the PPI network of the DEGs. Based on information from the STRING database, a PPI network was constructed to identify the most momentous proteins that may serve crucial roles in colorectal carcinogenesis and CRC development. A total of 1,012 nodes and 8,332 edges were contained in the PPI network (Fig. S1). Each gene was assigned a degree representing the number of neighbouring nodes in the network and changes in the expression of the proteins/genes. The top 10 nodes with the highest degrees in CRC were interleukin (IL)6, MYC, CDK1, cyclin (CCN)B1, CCNA2, DNA topoisomerase II $\alpha$, aurora kinase A, CXCL8, BUB1 mitotic checkpoint serine/threonine kinase and mitotic arrest deficient 2 like 1 (Table III). The high degrees of these genes indicated that their proteins may serve crucial roles in maintaining the whole protein interaction network. In addition, to explore the significance of these DEGs, centrality analysis of nodes in the PPI network was performed. The results demonstrated that IL6, MYC, NOTCH1, INHBA, CDK1, CCNB1 and CCNA2 were crucial genes (Table IV). As indicated in Fig. 1C, the expression levels of all of these crucial genes were markedly upregulated in CRC.

Validation of the expression levels of the crucial genes. To validate the expression levels of the crucial genes identified, UALCAN was employed based on TCGA data. The results suggested that these crucial genes were also significantly upregulated in CRC tissues, which was consistent with the microarray results (Fig. 4A). As presented in Fig. 4B, the protein expression levels of the crucial genes were markedly elevated in CRC tissues based on the HPA database, with the exception that INHBA protein expression data has not yet been made public.

Association between the expression levels of the crucial genes and survival. To determine the potential effect of the crucial genes on survival, UALCAN was further used to perform survival analyses based on TCGA data. The results indicated that elevated expression levels of INHBA significantly decreased survival probability of patients with CRC; however, upregulation of CCNB1 and CCNA2 expression were associated with increased survival rates (Fig. 5).

\section{Discussion}

With an increasing incidence rate and poor prognosis, CRC is commonly considered a devastating disease (2). By 2030, 2.2 million new cases and 1.1 million cases of mortality are predicted to occur, a situation that is responsible for the rising global burden of CRC (23). Although numerous studies have reported that unhealthy dietary habits, environmental changes and genetic aberrancies may be the primary causes of CRC, the precise molecular events orchestrating $\mathrm{CRC}$ initiation and progression remain elusive $(24,25)$. In the present study, bioinformatics methods were used to identify the crucial genes and pathways associated with CRC. A total of 1,085 DEGs, comprising 496 upregulated and 589 downregulated genes, 
Table II. GO enrichment analysis of the differentially expressed genes.

\begin{tabular}{|c|c|c|c|c|}
\hline Category & Term/gene function & Gene counts & Percentage & P-value \\
\hline \multirow[t]{5}{*}{ GOTERM_BP_DIRECT } & GO:0007067 mitotic nuclear division & 37 & 2.2 & $5.10 \times 10^{-7}$ \\
\hline & GO:0008284 positive regulation of cell proliferation & 53 & 3.2 & $7.25 \times 10^{-6}$ \\
\hline & GO:0006730 one-carbon metabolic process & 10 & 0.6 & $3.88 \times 10^{-5}$ \\
\hline & GO:0051301 cell division & 41 & 2.5 & $4.75 \times 10^{-5}$ \\
\hline & GO:0006955 immune response & 46 & 2.8 & $6.21 \times 10^{-5}$ \\
\hline \multirow[t]{5}{*}{ GOTERM_MF_DIRECT } & GO:0005179 hormone activity & 18 & 1.1 & $2.70 \times 10^{-5}$ \\
\hline & GO:0017080 sodium channel regulator activity & 10 & 0.6 & $6.21 \times 10^{-5}$ \\
\hline & GO:0051287 NAD binding & 10 & 0.6 & $2.63 \times 10^{-4}$ \\
\hline & GO:0042803 protein homodimerization activity & 66 & 4.1 & $4.18 \times 10^{-4}$ \\
\hline & GO:0005515 protein binding & 563 & 34.8 & $5.65 \times 10^{-4}$ \\
\hline \multirow[t]{5}{*}{ GOTERM_CC_DIRECT } & GO:0005615 extracellular space & 153 & 9.5 & $1.90 \times 10^{-16}$ \\
\hline & GO:0070062 extracellular exosome & 248 & 15.3 & $3.86 \times 10^{-13}$ \\
\hline & GO:0005576 extracellular region & 135 & 8.3 & $4.92 \times 10^{-6}$ \\
\hline & GO:0016324 apical plasma membrane & 37 & 2.3 & $1.08 \times 10^{-5}$ \\
\hline & GO:0005829 cytosol & 241 & 14.9 & $2.92 \times 10^{-5}$ \\
\hline
\end{tabular}

The top 5 GO terms in every category are presented. CC, cellular component; BP, biological processes; MF, molecular function; NAD, nicotinamide adenine dinucleotide; GO, Gene Ontology.

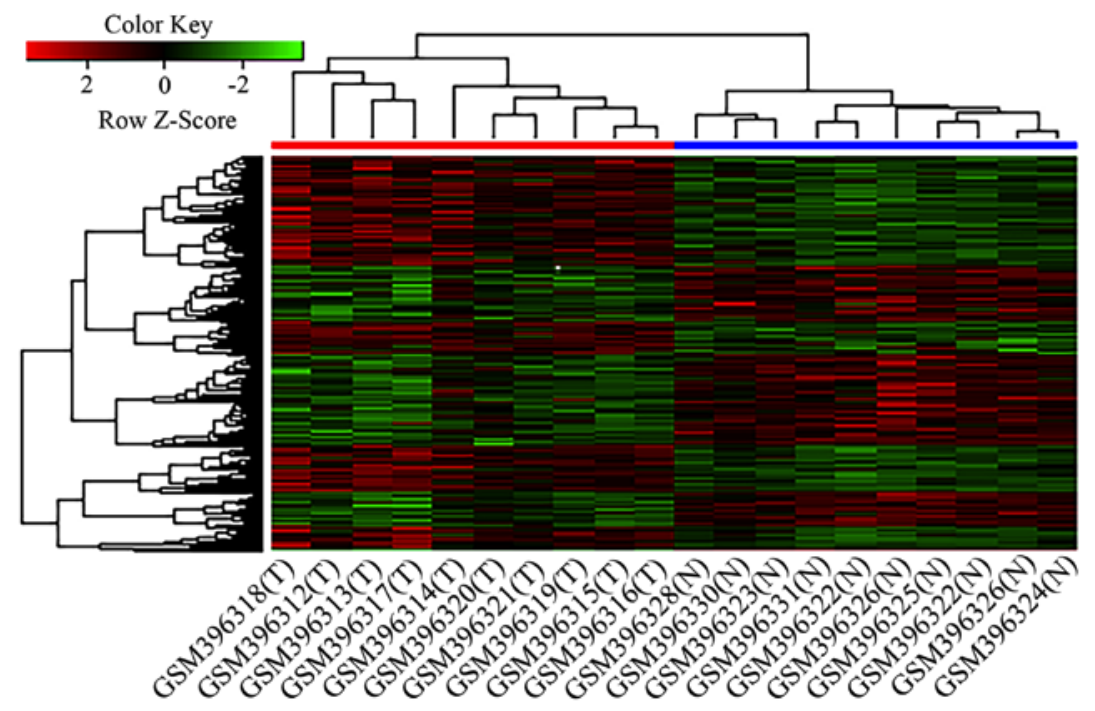

Figure 2. Hierarchical clustering analysis of the 1,085 differentially expressed genes. Red and green indicate upregulated and downregulated gene expression, respectively. T, tumor tissues; $\mathrm{N}$, normal tissues.

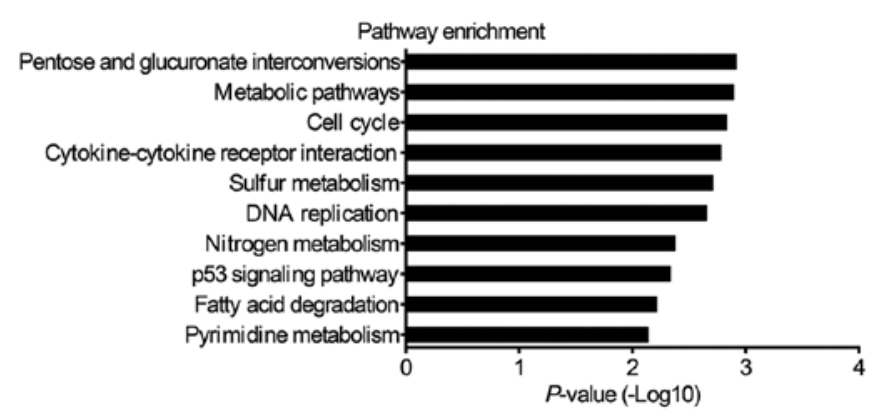

Figure 3. Top 10 most significantly enriched pathways for differentially expressed genes associated with colorectal cancer as determined by Kyoto Encyclopedia of Genes and Genomes pathway analysis. were identified in CRC tissues by comparison of gene expression profiles between 10 cancer tissues and corresponding non-cancerous tissues. Subsequently, hierarchical clustering analysis revealed that the DEGs exhibited distinct expression patterns between cancer and normal tissues.

To further comprehend the biological effects of the DEGs identified and the pathways associated with CRC that they accumulate in, GO term and KEGG pathway enrichment analyses were performed. The results of the GO term functional analysis indicated that the DEGs identified were mainly involved in the following terms: 'Mitotic nuclear division', 'positive regulation of cell proliferation', 'one-carbon metabolic process', 'cell division', ‘immune response', 'hormone 
Table III. Top 10 nodes with highest degrees of interaction in colorectal cancer.

\begin{tabular}{lccccc}
\hline Gene & Node degree & Betweenness centrality & Closeness centrality & Stress centrality & Clustering coefficient \\
\hline IL6 & 135 & 0.085 & 0.437 & 976,462 & 0.111 \\
MYC & 127 & 0.084 & 0.445 & $1,307,298$ & 0.127 \\
CDK1 & 118 & 0.021 & 0.397 & 354,950 & 0.387 \\
CCNB1 & 111 & 0.015 & 0.397 & 301,564 & 0.427 \\
CCNA2 & 102 & 0.009 & 0.387 & 200,222 & 0.475 \\
TOP2A & 102 & 0.012 & 0.397 & 236,116 & 0.496 \\
AURKA & 101 & 0.016 & 0.393 & 246,324 & 0.476 \\
CXCL8 & 97 & 0.029 & 0.416 & 408,590 & 0.202 \\
BUB1 & 94 & 0.005 & 0.369 & 106,422 & 0.567 \\
MAD2L1 & 94 & 0.009 & 0.366 & 135,590 & 0.530 \\
\hline
\end{tabular}

IL6, interleukin 6; CCNB1, cyclin B1; CCNA2, cyclin A2; TOP2A, DNA topoisomerase II ; AURKA, aurora kinase A; CXCL8, C-X-C motif chemokine ligand 8; BUB1, BUB1 mitotic checkpoint serine/threonine kinase; MAD2L1, mitotic arrest deficient 2 like 1.

Table IV. Top 3 genes ranked by the node centrality of the protein-protein interaction network.

\begin{tabular}{|c|c|c|c|c|c|c|c|c|}
\hline \multirow[b]{2}{*}{ Rank } & \multicolumn{2}{|c|}{ Degree centrality } & \multicolumn{2}{|c|}{ Betweenness centrality } & \multicolumn{2}{|c|}{ Closeness centrality } & \multicolumn{2}{|c|}{ Eigenvector centrality } \\
\hline & $\begin{array}{l}\text { Gene } \\
\text { symbol }\end{array}$ & $\begin{array}{l}\text { Expression } \\
\text { in CRC }\end{array}$ & $\begin{array}{l}\text { Gene } \\
\text { symbol }\end{array}$ & $\begin{array}{l}\text { Expression } \\
\text { in CRC }\end{array}$ & $\begin{array}{l}\text { Gene } \\
\text { symbol }\end{array}$ & $\begin{array}{l}\text { Expression } \\
\text { in CRC }\end{array}$ & $\begin{array}{l}\text { Gene } \\
\text { symbol }\end{array}$ & $\begin{array}{l}\text { Expression } \\
\text { in CRC }\end{array}$ \\
\hline 1 & IL6 & Upregulated & MYC & Upregulated & IL6 & Upregulated & CDK1 & Upregulated \\
\hline 2 & MYC & Upregulated & IL6 & Upregulated & MYC & Upregulated & CCNB1 & Upregulated \\
\hline 3 & NOTCH1 & Upregulated & INHBA & Upregulated & CDK1 & Upregulated & CCNA2 & Upregulated \\
\hline
\end{tabular}

CRC, colorectal cancer; IL6, interleukin 6; INHBA, inhibin $\beta$ A; CCNB1, cyclin B1; CCNA2, cyclin A2.

activity', 'sodium channel regulator activity', 'NAD binding', 'protein homodimerization activity' and 'protein binding', The results of the KEGG pathway analysis indicated that the DEGs were mainly enriched in metabolism, proliferation and and inflammatory response-related pathways, including 'Metabolic pathways', 'cell cycle', 'cytokine-cytokine receptor interaction', 'p53 signaling pathway' and 'pyrimidine metabolism'. Previous studies have demonstrated that the dysregulation of various BP terms, including 'cell division' and 'immune response', and the activation of multiple signalling pathway terms, including 'metabolic pathways', 'cell cycle' and 'p53 signalling', affect tumour development and patient survival (26-28). However, the underlying mechanisms through which the corresponding proteins in these signalling cascades promote tumorigenesis remain elusive. Therefore, further investigation of these identified BP and signalling pathway terms may aid in elucidating the underlying mechanisms of the carcinogenesis of CRC.

According to the node centrality of the PPI network, the seven crucial DEGs were identified as CDK1, CCNB1, CCNA2, IL6, MYC, INHBA and NOTCH1. Subsequently, the expression levels of these crucial genes were further investigated based on TCGA data. The results suggested that all crucial genes identified were significantly upregulated in colon carcinoma, which was consistent with the microarray results.
Among these crucial genes, CDK1, CCNB1, CCNA2, IL6 and NOTCH1 have been reported to be associated with CRC proliferation and progression (29-33). In particular, CDK1 is a key regulator of the $\mathrm{G}_{2} / \mathrm{M}$ checkpoint and is considered to be a possible target for cancer treatment (30). For instance, Thorenoor et al (29) indicated that CDK1 serves an important role in the regulation of the cell cycle and apoptosis of CRC cells through the 533 pathway. Fang et al (30) demonstrated that CCNB1 is positively associated with the expression of checkpoint kinase 1 , and is able to be activated by CDK1 to exert its oncogenic role in CRC cells. CCNA2, a novel oncogene, has been reported to serve a critical role in regulating cellular growth and apoptosis and may serve as a novel biomarker for diagnosis and therapy in CRC (31). IL6, a proinflammatory cytokine secreted by immune cells, may mediate immune and CRC cell cross-talk via miR-21 and miR-29b to produce an inflammatory microenvironment sufficient for promoting metastatic growth (32). In addition, Miteva et al (33) have reported that overexpression of IL6 promotes the migration and invasion of CRC cells, and upregulation of IL6 may be a transcriptional profile hallmark of colorectal metastases. Lv et al (34) have revealed that MYC regulates CRC progression, and that its overexpression enhances tumour metastasis and chemotherapy resistance in CRC. INHBA is a member of the transforming growth factor $\beta$ superfamily. Okano et al (35) 
A
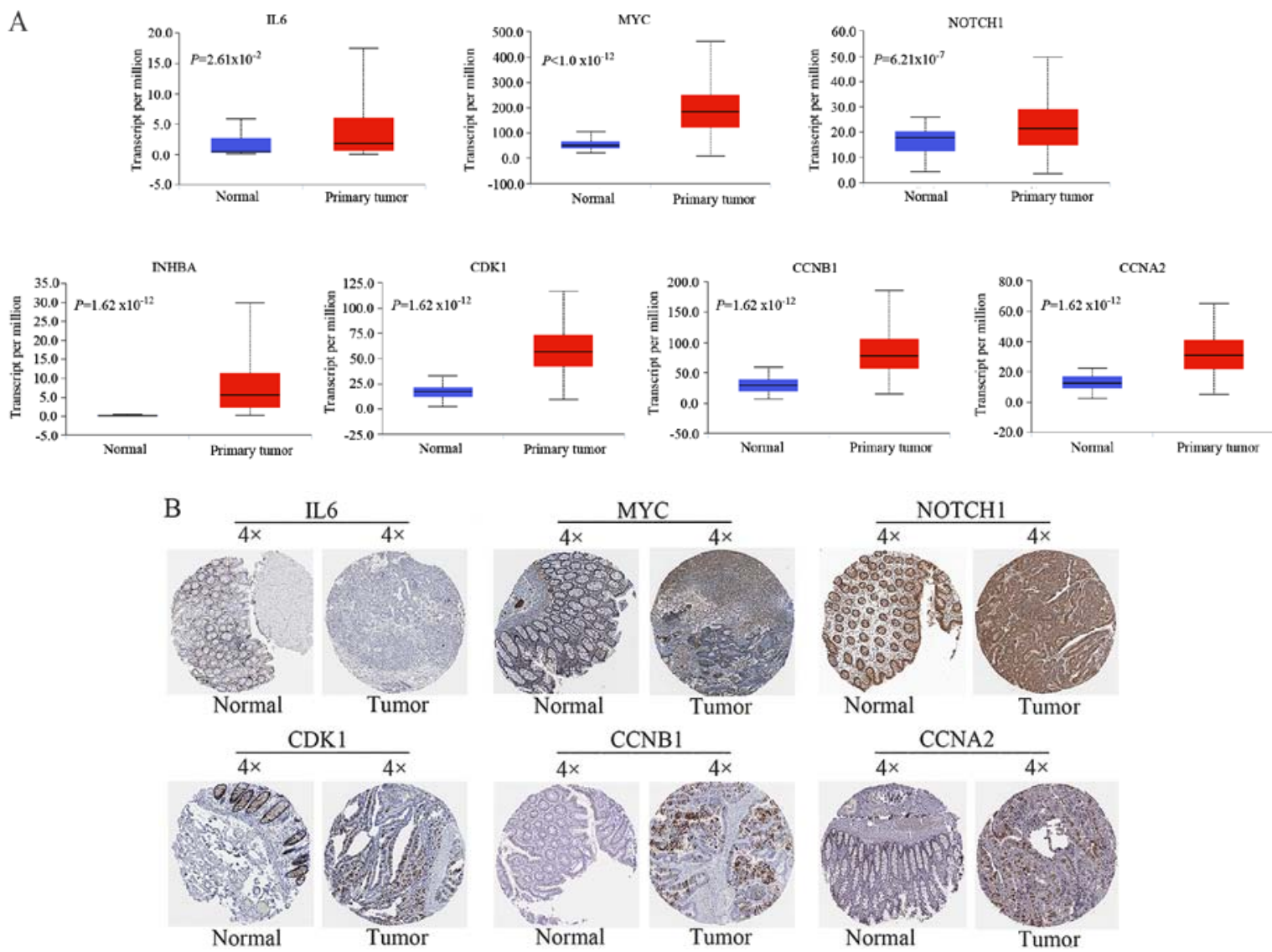

Figure 4. Crucial genes identified are upregulated in human CRC specimens. (A) Boxplots indicating the expression levels of the crucial genes in CRC tissues and normal tissues. (B) Immunohistochemical staining results (magnification, $\mathrm{x} 4$ ) for the protein expression levels of the crucial genes in normal tissues and CRC tissues. IL6, interleukin 6; INHBA, inhibin $\beta A$; CCNB1, cyclin B1; CCNA2, cyclin A2.

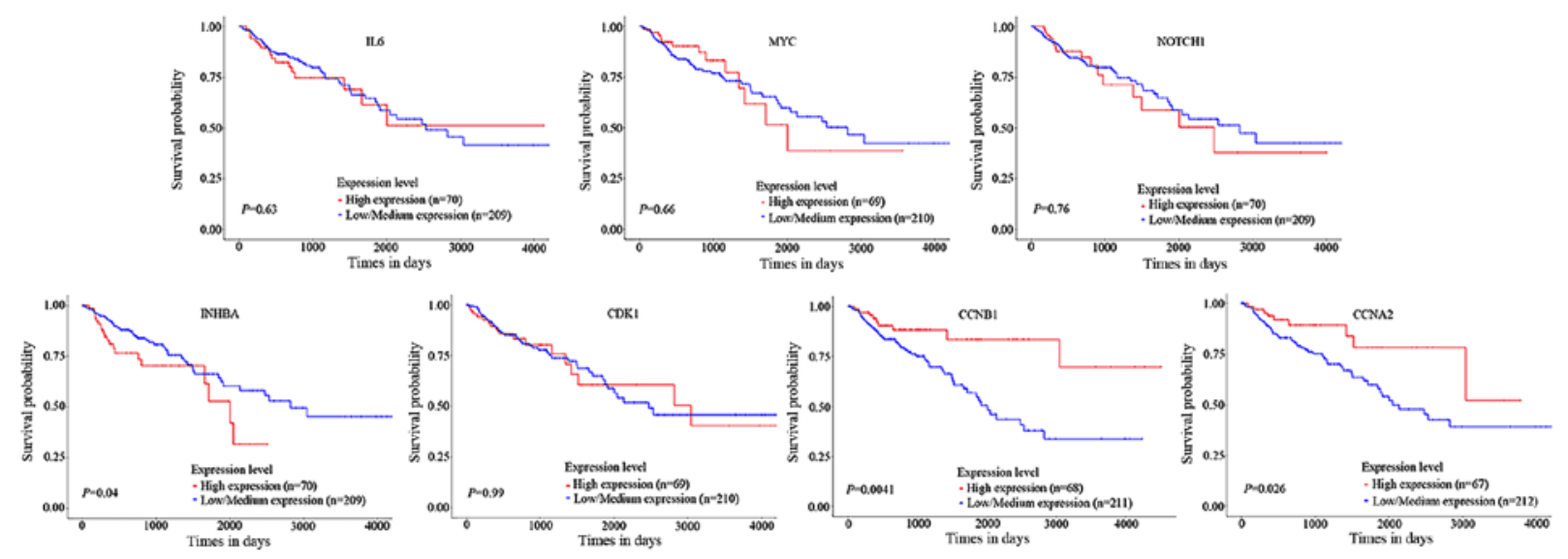

Figure 5. Kaplan-Meier plots for the association between the expression levels of the crucial genes and the overall survival of patients with CRC. IL6, interleukin 6; INHBA, inhibin $\beta$ A; CCNB1, cyclin B1; CCNA2, cyclin A2.

have suggested that overexpression of INHBA promotes cell growth and that its levels may be a useful prognostic marker for patients with CRC. Zhang et al (36) indicated that overexpression of NOTCH1 promotes the migration, invasion and proliferation of CRC cells. Furthermore, the NOTCH1 signalling pathway has been reported to mediate the radio- and chemoresistance of multiple tumour types $(37,38)$. For instance, inhibition of the NOTCH1 signalling pathway improves the radiosensitivity of CRC cells (36), providing a potential therapeutic target to improve the effect of radiotherapy in patients with CRC. To explore prognostic biomarkers for CRC, UALCAN was utilized to analyse the effect of the expression levels of certain crucial genes identified on the survival of patients with CRC. High expression levels of CCNB1, CCNA2 and INHBA were indicated to be associated with poor survival in patients with CRC.

In conclusion, the present bioinformatics study identified crucial genes and pathways associated with CRC, which will not 
only contribute to the elucidation of the pathogenesis of $\mathrm{CRC}$, but also provide potential prognostic markers and therapeutic targets for CRC management. However, the present study is limited partly due to the small quantity of samples and lack of experimental confirmation. Therefore, further verification of the expression profiles in CRC via in vivo and in vitro experiments is required.

\section{Acknowledgements}

The authors would like to thank Dr Yizhou Jiang for providing technical support.

\section{Funding}

No funding was received.

\section{Availability of data and materials}

The datasets used and/or analyzed during the current study are available from the corresponding author on reasonable request.

\section{Authors' contributions}

XQL, XDL and TQ conceived and designed the study. XQL, XDL and WC analyzed the microarray datasets and interpreted the results. XQL, XDL and WC drafted and revised the manuscript. All authors read and approved the final manuscript.

\section{Ethics approval and consent to participate}

Not applicable.

\section{Patient consent for publication}

Not applicable.

\section{Competing interests}

The authors declare that they have no competing interests.

\section{References}

1. Siegel RL, Miller KD and Jemal A: Cancer statistics, 2019. CA Cancer J Clin 69: 7-34, 2019.

2. Bray F, Ferlay J, Soerjomataram I, Siegel RL, Torre LA and Jemal A: Global cancer statistics 2018: GLOBOCAN estimates of incidence and mortality worldwide for 36 cancers in 185 countries. CA Cancer J Clin 68: 394-424, 2018.

3. Al Bandar MH and Kim NK: Current status and future perspectives on treatment of liver metastasis in colorectal cancer (Review). Oncol Rep 37: 2553-2564, 2017.

4. Kim SC, Shin YK, Kim YA, Jang SG and Ku JL: Identification of genes inducing resistance to ionizing radiation in human rectal cancer cell lines: Re-sensitization of radio-resistant rectal cancer cells through down regulating NDRG1. BMC Cancer 18: 594, 2018.

5. Andersen SE, Andersen IB, Jensen BV, Pfeiffer P, Ota T and Larsen JS: A systematic review of observational studies of trifluridine/tipiracil (TAS-102) for metastatic colorectal cancer. Acta Oncol 58: 1149-1157, 2019.

6. Jin S, Mu Y, Wang X, Liu Z, Wan L, Xiong Y, Zhang Y, Zhou L and $\mathrm{Li} \mathrm{L}$ : Overexpressed RACK1 is positively correlated with malignant degree of human colorectal carcinoma. Mol Bio Rep 41: 3393-3399, 2014.

7. Li XY, Hu Y, Li NS, Wan JH, Zhu Y and Lu NH: RACK1 acts as a potential tumor promoter in colorectal cancer. Gastroenterol Res Pract 2019: 5625026, 2019.
8. Ouyang S, Zhou X, Chen Z, Wang M, Zheng X and Xie M: LncRNA BCAR4, targeting to miR-665/STAT3 signaling, maintains cancer stem cells stemness and promotes tumorigenicity in colorectal cancer. Cancer Cell Int 19: 72, 2019.

9. Shangguan H, Tan SY and Zhang JR: Bioinformatics analysis of gene expression profiles in hepatocellular carcinoma. Eur Rev Med Pharmacol Sci 19: 2054-2061, 2015.

10. Kosti A, Harry Chen HI, Mohan S, Liang S, Chen Y and Habib SL: Microarray profile of human kidney from diabetes, renal cell carcinoma and renal cell carcinoma with diabetes. Genes Cancer 6: 62-70, 2015.

11. Christgen M, Geffers R, Kreipe H and Lehmann U: IPH-926 lobular breast cancer cells are triple-negative but their microarray profile uncovers a luminal subtype. Cancer Sci 104: 1726-1730, 2013.

12. Yu J, Li X, Zhong C, Li D, Zhai X, Hu W, Guo C, Yuan Y and Zheng S: High-throughput proteomics integrated with gene microarray for discovery of colorectal cancer potential biomarkers. Oncotarget 7: 75279-75292, 2016.

13. Shen X, Yue M, Meng F, Zhu J, Zhu X and Jiang Y: Microarray analysis of differentially-expressed genes and linker genes associated with the molecular mechanism of colorectal cancer. Oncol Lett 12: 3250-3258, 2016.

14. Snipstad K, Fenton CG, Kjaeve J, Cui G, Anderssen E and Paulssen RH: New specific molecular targets for radio-chemotherapy of rectal cancer. Mol Oncol 4: 52-64, 2010.

15. Gautier L, Cope L, Bolstad BM and Irizarry RA: Affy-analysis of Affymetrix GeneChip data at the probe level. Bioinformatics 20: 307-315, 2004.

16. Hardcastle TJ: Generalised empirical Bayesian methods for discovery of differential data in high-throughput biology. Bioinformatics 32: 195-202, 2016.

17. Huang DW, Sherman BT, Tan Q, Kir J, Liu D, Bryant D, Guo Y, Stephens R, Baseler MW, Lane HC and Lempicki RA: DAVID Bioinformatics Resources: Expanded annotation database and novel algorithms to better extract biology from large gene lists. Nucleic Acids Res 35: W169-W175, 2007.

18. Szklarczyk D, Morris JH, Cook H, Kuhn M, Wyder S and Simonovic M, Santos A, Doncheva NT, Roth A, Bork P, et al: The STRING database in 2017: Quality-controlled protein-protein association networks, made broadly accessible. Nucleic Acids Res 45: D362-D368, 2017.

19. Shannon P, Markiel A, Ozier O, Baliga NS, Wang JT, Ramage D, Amin N, Schwikowski B and Ideker T: Cytoscape: A software environment for integrated models of biomolecular interaction networks. Genome Res 13: 2498-2504, 2003.

20. Tang Y, Li M, Wang J, Pan Y and Wu FX: CytoNCA: A cytoscape plugin for centrality analysis and evaluation of protein interaction networks. Biosystems 127: 67-72, 2015.

21. Chandrashekar DS, Bashel B, Balasubramanya SAH, Creighton CJ, Ponce-Rodriguez I, Chakravarthi BVSK and Varambally S: UALCAN: A portal for facilitating tumor subgroup gene expression and survival analyses. Neoplasia 19: 649-658, 2017

22. Gao X, Wang X and Zhang S: Bioinformatics identification of crucial genes and pathways associated with hepatocellular carcinoma. Biosci Rep 38: BSR20181441, 2018.

23. Hassanain M, Al-Alem F, Simoneau E, Traiki TA, Alsaif F, Alsharabi A, Al-Faris $\mathrm{H}$ and Al-Saleh K: Colorectal cancer liver metastasis trends in the kingdom of Saudi Arabia. Saudi J Gastroenterol 22: 370-374, 2016.

24. Katsidzira L, Ocvirk S, Wilson A, Li J, Mahachi CB, Soni D, DeLany J, Nicholson JK, Zoetendal EG and O'Keefe SJD: Differences in fecal gut microbiota, short-chain fatty acids and bile acids link colorectal cancer risk to dietary changes associated with urbanization among zimbabweans. Nutr Cancer 71: 1313-1324, 2019.

25. Park Y, Park SJ, Cheon JH, Kim WH and Kim TI: Association of family history with cancer recurrence, survival, and the incidence of colorectal adenoma in patients with colorectal cancer. J Cancer Prev 24: 1-10, 2019.

26. Cordier-Bussat M, Thibert C, Sujobert P, Genestier L, Fontaine É and Billaud M: Even the Warburg effect can be oxidized: Metabolic cooperation and tumor development. Med Sci (Paris) 34: 701-708, 2018 (In French).

27. Subramanian $C$ and Cohen MS: Over expression of DNA damage and cell cycle dependent proteins are associated with poor survival in patients with adrenocortical carcinoma. Surgery 165: 202-210, 2019. 
28. Suehiro Y, Takemoto Y, Nishimoto A, Ueno K, Shirasawa B, Tanaka T, Kugimiya N, Suga A, Harada E and Hamano K: Dclk1 Inhibition cancels 5-FU-induced cell-cycle arrest and decreases cell survival in colorectal cancer. Anticancer Res 38: 6225-6230, 2018.

29. Thorenoor N, Faltejskova-Vychytilova P, Hombach S, Mlcochova J, Kretz M, Svoboda M and Slaby O: Long non-coding RNA ZFAS1 interacts with CDK1 and is involved in p53-dependent cell cycle control and apoptosis in colorectal cancer. Oncotarget 7: 622-637, 2016.

30. Fang Y, Yu H, Liang X, Xu J and Cai X: Chk1-induced CCNB1 overexpression promotes cell proliferation and tumor growth in human colorectal cancer. Cancer Biol Ther 15: 1268-1279, 2014.

31. Gan Y, Li Y, Li T, Shu G and Yin G: CCNA2 acts as a novel biomarker in regulating the growth and apoptosis of colorectal cancer. Cancer Manag Res 10: 5113-5124, 2018.

32. Patel SA and Gooderham NJ: IL6 mediates immune and colorectal cancer cell cross-talk via miR-21 and miR-29b. Mol Cancer Res 13: 1502-1508, 2015.

33. Miteva LD, Stanilov NS, Cirovski GM and Stanilova SA Upregulation of Treg-related genes in addition with IL6 showed the significant role for the distant metastasis in colorectal cancer. Cancer Microenviron 10: 69-76, 2017.

34. Lv Z, Wei J, You W, Wang R, Shang J, Xiong Y, Yang H, Yang X and $\mathrm{Fu} \mathrm{Z}$ : Disruption of the c-Myc/miR-200b-3p/PRDX2 regulatory loop enhances tumor metastasis and chemotherapeutic resistance in colorectal cancer. J Transl Med 15: 257, 2017.
35. Okano M, YamamotoH,OhkumaH,Kano Y,Kim H,Nishikawa S, Konno M, Kawamoto K, Haraguchi N, Takemasa I, et al: Significance of INHBA expression in human colorectal cancer. Oncol Rep 30: 2903-2908, 2013.

36. Zhang H, Jiang H, Chen L, Liu J, Hu X and Zhang H: Inhibition of Notch1/Hes1 signaling pathway improves radiosensitivity of colorectal cancer cells. Eur J Pharmacol 818: 364-370, 2018.

37. Sajadimajd S and Yazdanparast R: Differential behaviors of trastuzumab-sensitive and -resistant SKBR3 cells treated with menadione reveal the involvement of Notch1/Akt/FOXO1 signaling elements. Mol Cell Biochem 408: 89-102, 2015.

38. Prabakaran DS, Muthusami S, Sivaraman T, Yu JR and Park WY: Silencing of FTS increases radiosensitivity by blocking radiation-induced Notch1 activation and spheroid formation in cervical cancer cells. Int J Biol Macromol 126: $1318-1325,2019$.

(i) $(-)$ This work is licensed under a Creative Commons Attribution-NonCommercial-NoDerivatives 4.0 International (CC BY-NC-ND 4.0) License. 